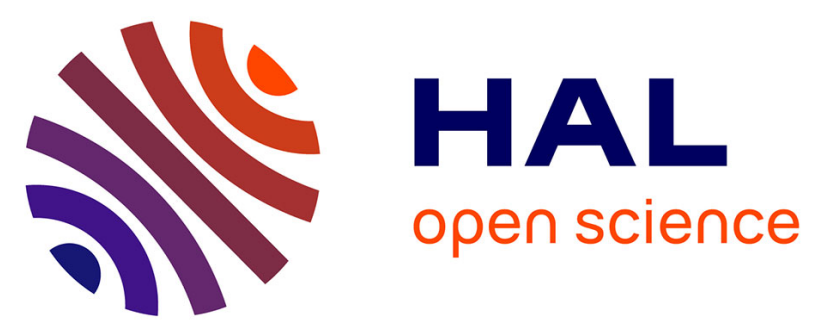

\title{
Symptomatologie dépressive à l'adolescence : rôle de la personnalité et de la conscience émotionnelle
}

Vincent Bréjard, Agnes Bonnet, Aurélie Pasquier, Jean-Louis Pedinielli

\section{To cite this version:}

Vincent Bréjard, Agnes Bonnet, Aurélie Pasquier, Jean-Louis Pedinielli. Symptomatologie dépressive à l'adolescence : rôle de la personnalité et de la conscience émotionnelle. Neuropsychiatrie de l'Enfance et de l'Adolescence, 2011, 59 (3), pp.157-162. 10.1016/j.neurenf.2010.07.013 . hal-01273518

\section{HAL Id: hal-01273518 \\ https://hal-amu.archives-ouvertes.fr/hal-01273518}

Submitted on 12 Feb 2016

HAL is a multi-disciplinary open access archive for the deposit and dissemination of scientific research documents, whether they are published or not. The documents may come from teaching and research institutions in France or abroad, or from public or private research centers.
L'archive ouverte pluridisciplinaire HAL, est destinée au dépôt et à la diffusion de documents scientifiques de niveau recherche, publiés ou non, émanant des établissements d'enseignement et de recherche français ou étrangers, des laboratoires publics ou privés. 
Symptomatologie dépressive à l'adolescence : rôle de la personnalité et de la conscience émotionnelle

Depressive symptoms in adolescence: Role of personality and emotion awareness

\section{Bréjard ${ }^{a *}$, A. Bonnet ${ }^{b}$, A. Pasquier ${ }^{c}, J_{-L}$ Pedinielli $^{\mathrm{b}}$}

${ }^{a}$ Laboratoire LabECD, EA 3259, UFR Psychologie, Université de Nantes-France

${ }^{\mathrm{b}}$ Laboratoire LPCP, EA 3278, UFR Psychologie-Sciences de l'éducation, Université de Provence-

France

${ }^{\mathrm{c}}$ Laboratoire PsyCLE, EA 3273, IUFM d'Aix-Marseille, Université de Provence-France

Article publié en mai 2011 dans la revue Neuropsychiatrie de l'enfance et de l'adolescence (vol. 59, n³, p. 157-162) 


\section{Résumé}

But de l'étude. L'objectif de cette étude était d'étudier les contributions relatives de dimensions de personnalité en référence au modèle de Cloninger et de la conscience émotionnelle à l'intensité de la symptomatologie dépressive, dans un échantillon communautaire d'adolescents. Cette recherche se proposait également d'évaluer l'effet médiateur du niveau de conscience émotionnelle sur la relation entre personnalité et dépression.

Patients et méthode. Un échantillon de 372 adolescents de collèges et lycées a été constitué. Les adolescents ont complété les questionnaires suivants: le Questionnaire Tridimensionnel de Personnalité (TPQ), l'Echelle de Niveau de Conscience Emotionnelle (LEAS), et l'échelle d'évaluation de la Dépression du Centre d'Etudes Epidémiologiques (CES-D)

Résultats. Les analyses en corrélations et en régressions hiérarchiques confirment l'existence de relations directes entre chacune des dimensions de personnalité et la dépression ainsi que l'effet médiateur partiel du niveau de conscience émotionnelle, mais uniquement dans le cas des dimensions « recherche de nouveauté » et « évitement du danger ».

Conclusion. Les résultats obtenus mettent en évidence le lien déjà observé dans des travaux antérieurs entre conscience émotionnelle et dépression d'une part, et entre personnalité et dépression d'autre part. Ils soulignent le rôle majeur joué par la personnalité et le fonctionnement émotionnel dans la vulnérabilité à présenter des symptômes dépressifs. L'importance de prendre en compte et d'évaluer le fonctionnement émotionnel dans la prise en charge de ces troubles à l'adolescence est discutée.

Mots-clés : Adolescence-dépression-personnalité-conscience émotionnelle

\section{Abstract}

Aim of the study. The aim of the study was to analyze the relating contribution of personality dimensions referring to Cloninger's model and emotional awareness to depressive symptoms intensity, in a community sample of adolescents. Our purpose was also to investigate the mediating role of the emotional awareness on the personality-depression relationship.

Method. A sample of 372 school students completed the following questionnaires: the Tridimensional Personality Questionnaire (TPQ), the Level of Emotional Awareness Scale (LEAS), and the Center for Epidemiologic Studies Depression scale (CES-D).

Results. Correlationnal and multiple hierarchical regressions analysis confirmed the existence of direct links between each personality dimensions and depression, and the partial mediating effect of emotional awareness, but only for the novelty seeking and the harm avoidance dimensions.

Conclusion. Our results confirm the relationships observed in previous research between emotion awareness and depression on one hand, and between personality and depression on the other hand. They highlight the major role played by personality and emotional processing in the vulnerability to 
present depressive symptoms. The importance of taking in account and assess the emotional processing in the adolescent's depression clinical support is discussed.

Key-words: Adolescence-depression-personality-emotional awareness 


\section{Introduction}

Les troubles de l'humeur à l'adolescence sont une des pathologies psychiques les plus répandues pour cette tranche d'âge. Sur le plan épidémiologique, la prévalence du trouble est de 5 à 7,5\% en fonction des études [1]. De plus, on retrouve une part importante de comorbidité tous sexes confondus (17 à $30 \%$ selon les études) avec d'autres troubles fréquents à l'adolescence tels que la consommation de substances psychoactives, les troubles externalisés (troubles des conduites, trouble oppositionnel) et les conduites à risques [2-4]. La problématique des troubles de l'humeur à l'adolescence constitue donc un véritable problème de santé publique.

De nombreux facteurs semblent impliqués dans la vulnérabilité aux troubles dépressifs chez les adolescents tels que l'environnement familial (décès d'un parent, divorce), les évènements de vie négatifs [5], le développement pubertaire, les dimensions de personnalité ou le fonctionnement émotionnel. Dans une perspective psychopathologique, les relations entre personnalité et dépression ont fait l'objet de différents travaux. Parmi les modèles de personnalité, celui de Cloninger a démontré sa pertinence dans l'étude de facteurs de vulnérabilité aux troubles dépressifs. Ainsi dans une étude utilisant le TCI et la CES-D avec un échantillon de 804 sujets, l'évitement du danger est lié au score total de dépression, alors que la dépendance à la récompense associée à la persistance est associée aux symptômes somatiques ainsi qu'à la perte d'appétit et à la fatigue. La recherche de nouveauté est quant à elle associée négativement avec la dépression [6]. Hansenne et al. [7] observent que l'augmentation du niveau de la dépression est positivement corrélé avec le score d'évitement du danger, et l'interprètent comme une instabilité de cette dimension qui est modifiée par la présence d'un trouble thymique.

Une autre partie des travaux sur la dépression s'est intéressé au rôle du fonctionnement émotionnel, dans une perspective dimensionnelle ou processuelle. Ainsi, a été mise en évidence l'influence de certaines dispositions stables dans le fonctionnement émotionnel, entraînant des biais dans la perception de l'environnement. Ces dispositions stables, ou émotions-traits, sont conçues comme des prédispositions à éprouver des émotions de type plutôt positif ou négatif [8]. Watson et Clark émettent ainsi l'hypothèse que les individus qui ont un score élevé sur une dimension de personnalité appelée «émotionnalité négative » (NA : Negative Affect) auront une prédisposition à éprouver préférentiellement des émotions négatives et que cette tendance aura un effet favorisant la survenue de troubles dépressifs ou 
anxieux [9]. La symptomatologie dépressive apparaît donc liée à la prédisposition à éprouver des émotions négatives, ainsi qu'à la tendance à produire des interprétations négatives de l'environnement. Cette relation a été retrouvée dans une étude australienne portant sur un échantillon de 780 adolescents, qui a confirmé la pertinence de l'émotionnalité négative comme facteur explicatif des troubles dépressifs [10].

Un deuxième groupe de travaux s'est intéressé à l'influence des troubles émotionnels, et en particulier de la régulation des émotions. Parmi ceux-ci, l'anhédonie et l'alexithymie ont fait l'objet de nombreuses contributions, et ont été identifiées comme des facteurs en forte relation avec la dépression. De nombreux travaux ont apporté la preuve de l'existence d'une relation significative entre alexithymie et dépression, tant dans la population générale que sur des échantillons pathologiques [11-13].

Plus globalement, on peut observer que le fonctionnement émotionnel intervient de différentes manières dans la dépression. D'un point de vue dispositionnel, il constitue un facteur de vulnérabilité dans le cas d'une tendance à ressentir de façon privilégiée des émotions négatives. D'un point de vue dynamique, il joue un rôle important par les incidences sur la dépression entraînant des difficultés dans le traitement de l'information émotionnelle.

Il existe peu de travaux mettant en relation fonctionnement émotionnel, personnalité et dépression. Une étude de Watson et al. [14] montre cependant que l'humeur dépressive est corrélée aux deux dimensions de l'extraversion et de l'émotionnalité positive, elles-mêmes liées à l'anhédonie et à l'anxiété sociale. Les deux dimensions du névrosisme et de l'affectivité négative sont reliées plus fortement à la dysphorie et à la détresse subjective, tout en prédisant la présence de troubles psychopathologiques, mais de manière non spécifique.

\section{Hypothèses}

Suite aux différents constats issus de ces travaux, nous avons formulé les hypothèses suivantes :

H1 : Il existe une relation significative entre dimensions de personnalité et dépression, ainsi qu'entre niveau de conscience émotionnelle et dépression

H2 : Les dimensions de personnalité « recherche de nouveauté », «évitement du danger, » « dépendance à la récompense » associées au niveau de conscience émotionnelle constituent des prédicteurs significatifs des symptômes dépressifs 


\section{Méthode}

\subsection{Participants}

Nous avons constitué un échantillon d'adolescents issus de collèges et lycées de filières générales et professionnelles. Sur l'ensemble des adolescents rencontrés, 372 ont donné leur accord pour participer à l'étude. Ils étaient âgés de 12 à 19 ans (âge moyen $=14$ ans et 10 mois \pm 1.42$)$, et se répartissaient en 204 filles $(54,8 \%)$ et 168 garçons $(45,2 \%)$. Pour $58 \%$ des adolescents, les parents étaient mariés, alors que 36\% vivaient en famille monoparentale.

\subsection{Mesures}

Les participants ont complété un questionnaire de données sociodémographiques, ainsi que trois questionnaires : l'évaluation de la dépression a été réalisée par l'échelle de dépression du Centre d'études épidémiologiques (CES-D) [15]. Ses propriétés psychométriques ont été étudiées avec un échantillon d'adolescents, avec des résultats satisfaisants $(\alpha=0.85)$ que de la sensibilité [16].

Les traits de personnalité ont été mesurés avec le questionnaire tridimensionnel de la personnalité (TPQ) [17] qui évalue les quatre dimensions du tempérament que sont la Recherche de Nouveauté (RDN), l'Evitement du Danger (ED), la Dépendance à la Récompense (DR) et la Persistance (P), conformément au modèle de Cloninger [18]. Utilisé dans plusieurs études auprès d'adolescents, le TPQ a permis de proposer une évaluation satisfaisante de la personnalité, notamment dans le cadre de recherches sur les conduites à risques et la dépression [19-21]

Le niveau de conscience émotionnelle a été évalué avec la LEAS. Cet instrument d'évaluation consiste en 20 items dans lesquels on demande au sujet de se représenter dans une courte scène le mettant en interaction avec une autre personne. Le sujet doit répondre à deux questions : l'une porte sur ce qu'il ressentirait dans la situation, l'autre porte sur ce que l'autre personne ressentirait, en commençant la phrase par «je me sentirais ou je ressentirais... » et «l'autre personne ressentirait ou se sentirait.... ». La cotation s'effectue grâce à une grille de cotation de 0 à 5 et permet d'obtenir un score de 0 à 80 . La LEAS comporte deux sous-échelles évaluant les capacités du sujet à être conscient de ses états émotionnels, ainsi que ses capacités à les conceptualiser chez autrui. La structure factorielle de l'échelle, ainsi que ses qualités psychométriques, sont satisfaisantes [22, 23]. Nous avons 
été amenés à modifier certains items du fait de leur formulation (ex : situation professionnelle décrite faisant référence à un «patron ») [24].

\subsection{Procédure}

Nous avons demandé l'accord préalable des chefs d'établissement, des parents et des adolescents. Le recueil des données s'est effectué anonymement après que le consentement écrit des participants ait été obtenu. Les passations ont été réalisées collectivement sous la supervision des investigateurs de l'étude ou des surveillants des établissements.

\subsection{Démarche d'analyse statistique}

Nous avons procédé à une analyse de corrélations bivariées puis réalisé deux régressions afin d'évaluer l'effet médiateur du niveau de conscience émotionnelle, conformément aux recommandations de Baron et Kenny [25]. Pour cela, une régression multiple testant les liens entre dimensions de personnalité et niveau de conscience émotionnelle, et une régression hiérarchique avec un premier modèle incluant les quatre dimensions du tempérament, puis un second avec ces mêmes dimensions auxquelles a été ajouté le niveau de conscience émotionnelle ont été réalisées. Une médiation pourra être identifiée si a) la variable explicative (les dimensions de personnalité) contribue de manière significative à la variance du médiateur (le niveau de conscience émotionnelle) b) la variable explicative (les dimensions de personnalité) contribue de manière significative à la variance de la variable « cible » (la dépression) c) l'effet de la variable explicative diminue après introduction du médiateur dans l'équation de régression. La contribution respective de chaque prédicteur est matérialisée par l'indice $\beta$ et la statistique $p$. Les traitements statistiques ont été réalisés grâce au logiciel SPSS 11.5 (SAS inc).

\section{Résultats}

Le tableau 1 présente les statistiques descriptives ainsi que les différences intersexes. On observe ainsi que l'âge moyen des garçons est significativement différent de celui des filles. De même, il existe une différence liée au sexe à la dimension Dépendance à la Récompense (DAR) et au score de dépression. Dans les deux cas, les filles présentent un niveau de DAR et de dépression supérieur aux garçons. Les corrélations entre les variables sont présentées dans le tableau 2. 
On observe des corrélations modérées ( $r>a ̀$ 0,30) entre dimensions de personnalité et dépression, ainsi qu'entre niveau de conscience émotionnelle global et dépression. Ainsi, un niveau élevé de dépression sera associé à une faible Recherche de Nouveauté (RDN), un fort Evitement Du Danger (EDD), une forte Persistance (P), et à un faible Niveau de Conscience Emotionnelle (NCE). On observe également des corrélations plus faibles $(0,10<r<0,30)$ mais néanmoins significatives entre RDN, EDD et NCE, respectivement négative dans le cas de la RDN et positive dans le cas de l'EDD.

Concernant les analyses de régression multiples, le premier modèle, avec comme critères les dimensions de personnalité, explique $9 \%$ de la variance du niveau de conscience émotionnel, avec comme critères significatifs uniquement la RDN et l'EDD. Dans le second modèle testant une régression hiérarchique, les variables démographiques ont été introduites en premier, mais la part de variance expliquée restait modeste (3\%). Puis les dimensions de personnalité ont été introduites, augmentant la part de variance expliquée à $38 \%$. Enfin, le niveau de conscience émotionnelle a été ajouté dans un troisième temps, le modèle final expliquant $48 \%$ de la variance du score total de dépression, mais avec une diminution de la contribution respective des dimensions RDN et HA.

\section{Discussion}

Notre objectif dans cette étude était d'analyser les contributions relatives de différentes dimensions de personnalité et du niveau de conscience émotionnelle, ainsi que le potentiel effet médiateur du NCE sur la personnalité. Les résultats obtenus valident partiellement les hypothèses formulées. En effet, conformément à des travaux antérieurs chez l'adulte [26], la dépression chez les adolescents semble négativement liée à la tendance à l'activation comportementale (représentée par la $\mathrm{RDN}$ ) et à la tendance à l'inhibition comportementale (représentée par l'EDD). Elle n'est en revanche pas liée à la sensibilité aux renforcements de l'environnement (représentée par la DAR), ni à la tendance à persévérer dans un comportement -représentée par la P). On peut l'expliquer par la spécificité de la période adolescente, où l'autonomisation constitue un enjeu majeur du développement, amenant les adolescents à tenter de se représenter comme moins dépendants d'autrui.

La dépression est également liée négativement au niveau général de développement cognitivo-émotionnel, ainsi qu'à la sous-dimension intrasubjective de celui-ci. Nous n'avons pas retrouvé de relation significative entre la sous-dimension intersubjective et le niveau de dépression. Il apparaît ainsi qu'une augmentation de la capacité à expérimenter et mentaliser 
des états émotionnels subjectifs complexes est liée à une diminution de l'importance des symptômes dépressifs. Dans une recherche sur l'adolescent, des relations significatives ont été retrouvées entre alexithymie et expérience dépressive [27]. Néanmoins, le niveau de conscience émotionnelle ne se réduit pas à l'alexithymie, dont il peut, tout au plus constituer une explication potentielle [28]. Enfin, nous avons pu mettre en évidence l'existence d'un possible effet médiateur partiel du niveau de conscience émotionnelle sur le lien entre la dépression et deux dimensions de personnalité : la «recherche de nouveauté » et l' «évitement du danger ». Le niveau de conscience émotionnelle pourrait constituer une variable intermédiaire $\mathrm{du}$ fonctionnement psychique, influençant les relations entre dimensions fondamentales de la personnalité et expression symptomatique. Les adolescents affectés de signes dépressifs présenteraient un pattern d'inhibition cognitive et comportementale excessif, du fait d'une incapacité à accéder à des expériences émotionnelles subjectives complexes. Cette incapacité pourrait découler d'un fonctionnement défensif maintenant un faible niveau de conscience émotionnelle et évitant les émotions «complexes ». Les émotions ne joueraient alors plus leur rôle de «régulation» des comportements ni de processus adaptatif, amenant les adolescents à présenter un niveau significatif de dépression. Enfin, l'incapacité à se représenter de manière complexe les contenus émotionnels pourraient être liée à un arrêt développemental, du fait de l'environnement, à un moment de l'histoire infantile où l'inhibition était privilégiée, de même que la confrontation à des éprouvés subjectifs potentiellement traumatiques pour le sujet [29, 30].

Ces interprétations doivent prendre en considération plusieurs limites à cette étude. Premièrement, nous n'avons utilisé que des échelles d'auto-évaluation, en particulier de la symptomatologie dépressive. Même si la CES-D constitue un instrument de choix dans l'évaluation de la présence d'une symptomatologie dépressive, elle manque de spécificité et ne permet pas de différencier les syndromes dépressifs. L'utilisation d'un entretien semistructuré tel que le MINI, pourrait lever cette difficulté. Deuxièmement, le niveau intellectuel n'a pas été contrôlé. Or la LEAS faisant appel au lexique émotionnel, des différences pourraient être liées aux lacunes dans la connaissance de termes émotionnels complexes. Néanmoins, la capacité à expérimenter des états émotionnels complexes peut être liée justement à la complexité des informations stockées en mémoire sémantique. Ainsi l'expression verbale et/ou écrite pourrait constituer un indicateur représentatif de la 
conscience émotionnelle, surtout si elle était complétée par l'évaluation de biais cognitifs dans la reconnaissance de stimuli émotionnels par exemple.

Troisièmement, la construction transversale de l'étude ne permet pas de conclure avec certitude à des relations de causalité, qui demanderont à être confirmées par des études comparant enfants et adolescents, ou longitudinales.

\section{Conclusion}

Les résultats obtenus mettent en évidence le rôle central du fonctionnement émotionnel et de son niveau d'élaboration dans l'intensité de la symptomatologie dépressive, ainsi que son rôle transactionnel entre personnalité et symptôme. Ils soulignent l'intérêt d'une évaluation psychologique du fonctionnement émotionnel, ainsi que de son niveau de développement, dans la clinique des adolescents. Dans ce cadre, la construction de normes constitue un enjeu important de l'utilisation de la LEAS dans une perspective clinique. Par ailleurs, la capacité à expérimenter des états émotionnels complexes sans que ceux-ci ne deviennent déstabilisants pour l'adolescent déprimé s'inscrit dans le processus «adolescens ». Elle constitue un axe prépondérant du travail clinique psychothérapique.

\section{Conflit d'intérêt : aucun}




\section{Références}

[1] Braconnier A, Chiland C, Choquet M, Pomarede R: Dépression : adolescentes, adolescents. Paris: Bayard, 1995.

[2] Pesa JA, Cowdery JE, Westerfield RC, Wang M: Self-reported depression and risk-taking behaviors among Hispanic adolescents. Psychol Rep 1997; 81(1): 235-43.

[3] Kosunen E, Kaltiala-Heino R, Rimpela M, Laippala P: Risk-taking sexual behaviour and self-reported depression in middle adolescence--a school-based survey. Child Care Health Dev 2003; 29(5): 337-44.

[4] Khlat M, Sermet C, Le Pape A: Increased prevalence of depression, smoking, heavy drinking and use of psycho-active drugs among unemployed men in France. Eur J Epidemiol 2004; 19(5): 445-51.

[5] Flament M, Cohen D, Choquet M, Jeammet P, Ledoux S: Phenomenology, psychosocial correlates, and treatment seeking in major depression and dysthymia of adolescence. $\mathrm{J}$ Amer Acad Child Adolesc Psychiatry 2001; 40(9): 1070-1078.

[6] Grucza RA, Przybeck TR, Spitznagel EL, Cloninger CR: Personality and depressive symptoms: a multi-dimensional analysis. J Affect Disord 2003; 74(2): 123-30.

[7] Hansenne M, Pitchot W, Gonzalez Moreno A, Machurot PY, Ansseau M: The Tridimensional Personality Questionnaire (TPQ) and depression. Eur Psychiatry 1998; 13(2): 101-103.

[8] Watson D, Clark LA: Negative affectivity: the disposition to experience aversive emotional states. Psychol Bull 1984; 96(3): 465-90.

[9] Watson D, Clark LA: On traits and temperament: general and specific factors of emotional experience and their relation to the five-factor model. J Pers 1992; 60(2): 441-76.

[10] Boyd CP, Gullone E: An investigation of negative affectivity in Australian adolescents. J Clin Child Psychol 1997; 26(2): 190-7.

[11] Modestin J, Furrer R, Malti T: Study on alexithymia in adult non-patients. J Psychos Research 2004; 56(6): 707-709.

[12] Loas G, Otmani O, Lecercle C, Jouvent R: Relationships between the emotional and cognitive components of alexithymia and dependency in alcoholics. Psychiatr Research 2000; 96(1): 63-74.

[13] Honkalampi K, Hintikka J, Tanskanen A, Lehtonen J, Viinamaki H: Depression is strongly associated with alexithymia in the general population. J Psychos Research 2000; 48(1): 99-104.

[14] Watson D, Clark LA, Weber K, Assenheimer JS, Strauss ME, McCormick RA: Testing a tripartite model: II. Exploring the symptom structure of anxiety and depression in student, adult, and patient samples. J Abnorm Psychol 1995; 104(1): 15-25.

[15] Fuhrer T, Rouillon F: La version française de l'échelle CES-D (Center for Epidemiologic Studies-Depression Scale). Description et traduction de l'échelle d'autoévaluation. Psychiatr Psychobiologie 1989; 4: 163-166.

[16. Chabrol H, Montovany A, Chouicha K, Duconge E: Etude de la CES-D dans un échantillon de 1,953 adolescents scolarisés. Encephale 2002; 28(5 Pt 1): 429-32.

[17] Cloninger CR, Przybeck TR, Svrakic DM: The Tridimensional Personality Questionnaire: U.S. normative data. Psychol Rep 1991; 69(3 Pt 1): 1047-57.

[18] Cloninger CR, Svrakic DM, Przybeck TR: A psychobiological model of temperament and character. Arch Gen Psychiatry 1993; 50(12): 975-90.

[19] Wills TA, Vaccaro D, McNamara G: Novelty seeking, risk taking, and related constructs as predictors of adolescent substance use: An application of Cloninger's theory. J Subst Abuse 1994; 6(1): 1-20. 
[20] Wills TA, Windle M, Cleary SD: Temperament and novelty seeking in adolescent substance use: Convergence of dimensions of temperament with constructs from Cloninger's theory. J Pers Social Psychology 1998; 74(2): 387-406.

[21] Michel G, Mouren-Simeoni M-C, Perez-Diaz F: The Tridimensional Personality Questionnaire and the Sensation-Seeking scale in depressed adolescent: state versus trait issues. Eur Child Adol Psychiatry 1999; 8(2,S.379,): 126.

[22] Lane RD, Quinlan DM, Schwartz GE, Walker PA, Zeitlin SB: The Levels of Emotional Awareness Scale: a cognitive-developmental measure of emotion. J Pers Assess 1990; 55(12): $124-34$.

[23] Bydlowski S, Corcos M, Paterniti S, Guilbaud O, Jeammet P, Consoli SM: Validation de la version francaise de l'echelle des niveaux de conscience émotionnelle. Encephale 2002; 28(4): 310-20.

[24] Bréjard V, Bonnet A, Pedinielli JL: Développement cognitivo-émotionnel, régulation des émotions et comportements à risques : une étude exploratoire chez l'adolescent.

Neuropsychiatr Enfance Adolesc 2005 ; 53(8), 395-400.

[25] Baron RM, Kenny DA: The moderator-mediator variable distinction in social psychological research: Conceptual, strategic, and statistical considerations. J Pers Social Psychology 1986; 51(6): 1173-1182.

[26] Richter J, Polak T, Eisenmann M: Depressive mood and personality in terms of temperament and character among the normal population and depressive inpatients. Pers Ind Differences 2003; 35(4): 917-927.

[27] Farges F, Corcos M, Speranza M, et al.: Alexithymie et toxicomanie: Lien avec la dépression. Encephale 2004; 30(3): 201-211.

[28] Jouanne C, Edel Y, Carton S: Déficits émotionnels chez des patients polytoxicomanes. Ann Médico Psychologiques 2005; 163(8): 625-630.

[29] Stolorow RD: The Contextuality of Emotional Experience. Psychoanalytic Psychology 2005; 22(1): 101-106.

[30] Stolorow RD: Psychoanalysis of developmental arrests, 6 ed. Madison, Conn:

International University Press, 1989. 
Tableau 1. Statistiques descriptives et différences intersexes (t de Student)

\begin{tabular}{|c|c|c|c|c|c|c|c|c|}
\hline & \multicolumn{2}{|c|}{ Total $(n=372)$} & \multicolumn{2}{|c|}{ Garçons $(n=168)$} & \multicolumn{2}{|c|}{ Filles $(n=204)$} & \multirow[b]{2}{*}{$t(2,370)$} & \multirow[b]{2}{*}{$p$} \\
\hline & M & (ET) & M & (ET) & M & (ET) & & \\
\hline Age & 14,81 & $(1,42)$ & 15,26 & $(1,52)$ & 14,43 & $(1,21)$ & 5,81 & 0,00 \\
\hline RDN & 15,57 & $(4,31)$ & 15,63 & $(4,45)$ & 15,52 & $(4,21)$ & 0,25 & 0,80 \\
\hline EDD & 16,06 & $(5,34)$ & 15,53 & $(5,18)$ & 16,50 & $(5,44)$ & $-1,76$ & 0,08 \\
\hline DAR & 13,01 & $(3,02)$ & 12,33 & $(2,76)$ & 13,57 & $(3,12)$ & $-4,00$ & 0,00 \\
\hline $\mathrm{P}$ & 4,86 & $(1,97)$ & 4,82 & $(1,95)$ & 4,89 & $(1,98)$ & $-0,37$ & 0,71 \\
\hline LEAS Soi & 43,93 & $(6,20)$ & 43,55 & $(6,35)$ & 44,25 & $(6,06)$ & $-1,07$ & 0,28 \\
\hline LEAS Autrui & 41,15 & $(6,24)$ & 41,05 & $(5,97)$ & 41,23 & $(6,48)$ & $-0,27$ & 0,79 \\
\hline LEAS Global & 49,30 & $(5,63)$ & 48,97 & $(5,72)$ & 49,58 & $(5,56)$ & $-1,04$ & 0,30 \\
\hline CES-D & 18,09 & $(9,95)$ & 16,34 & $(7,97)$ & 19,53 & $(11,14)$ & $-3,12$ & 0,00 \\
\hline
\end{tabular}

M:Moyenne; ET: écart-type; RDN: Recherche de Nouveauté; EDD: Evitement Du Danger;

DAR: Dépendance A la Récompense; P: Persistance; LEAS: Niveau de Conscience Emotionnelle; CESD: Niveau de Dépression 
Tableau 2 Corrélations entre la dépression, les dimensions de personnalité et le niveau de conscience émotionnelle

\begin{tabular}{|c|c|c|c|c|c|c|c|c|c|}
\hline & 1 & 2 & 3 & 4 & 5 & 6 & 7 & 8 & 9 \\
\hline $1 \mathrm{Age}$ & 1 & $0,11 *$ & $-0,05$ & $-0,06$ & $-0,06$ & 0,04 & $-0,05$ & 0,07 & $-0,12 *$ \\
\hline $2 \mathrm{RDN}$ & & 1 & $-0,42 * *$ & 0,07 & $-0,35 * *$ & $-0,16^{* *}$ & $-0,04$ & $-0,21 * *$ & $-0,46^{* *}$ \\
\hline 3 EDD & & & 1 & $0,14 * *$ & $0,16^{* *}$ & $-0,22 * *$ & 0,02 & $0,26 * *$ & $0,48 * *$ \\
\hline $4 \mathrm{DAR}$ & & & & 1 & $-0,02$ & 0 & $-0,06$ & $-0,07$ & $0,2 * *$ \\
\hline $5 \mathrm{P}$ & & & & & 1 & $-0,06$ & 0,06 & $-0,1$ & $0,33 * *$ \\
\hline 6 LEAS Soi & & & & & & 1 & $0,56 * *$ & $0,86 * *$ & $-0,37 * *$ \\
\hline 7 LEAS Autrui & & & & & & & 1 & $0,67 * *$ & $-0,07$ \\
\hline 8 LEAS Global & & & & & & & & 1 & $-0,46^{* *}$ \\
\hline 9 CES-D & & & & & & & & & 1 \\
\hline
\end{tabular}

$* p<0,05 * * p<0,01$

RDN: Recherche de Nouveauté; EDD: Evitement Du Danger;

DAR: Dépendance A la Récompense; P: Persistance; LEAS: Niveau de Conscience Emotionnelle;

CESD: Score de dépression 
Tableau 3 : Régressions hiérarchiques et analyse de l'effet médiateur du niveau de conscience émotionnelle sur le lien entre personnalité et dépression

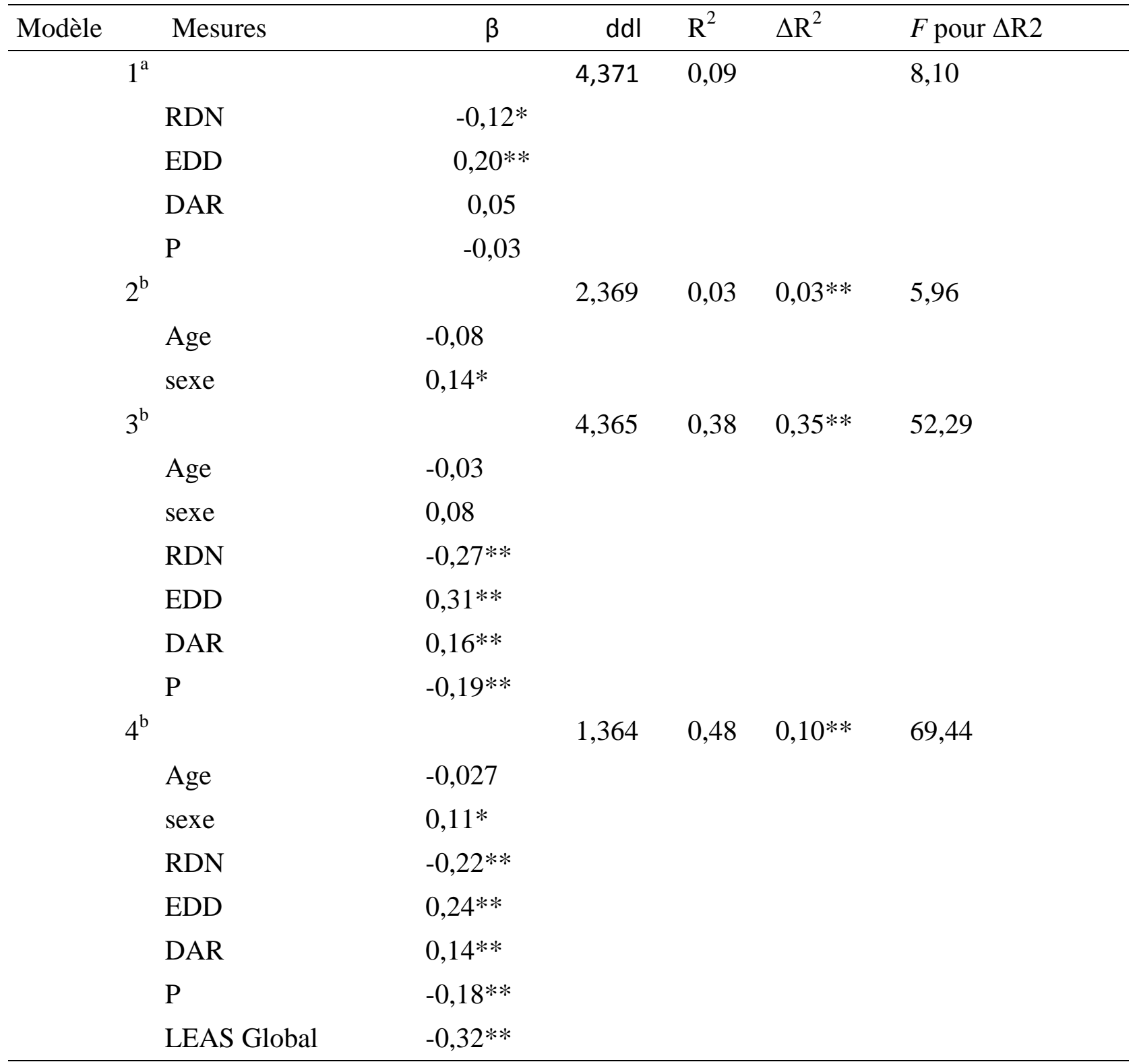

$* \mathrm{p}<.05 * * \mathrm{p}<.01$

RDN: Recherche de Nouveauté; EDD: Evitement Du Danger;

DAR: Déprendance A la Récompense; P: Persistance; LEAS: Niveau de Conscience Emotionnelle; CESD: Niveau de Dépression

${ }^{\mathrm{a}}$ Variable à expliquer: Niveau de Conscience Emotionnelle ${ }^{\mathrm{b}}$ Variable à expliquer: Dépression 